\title{
Properties of meromorphic solutions of Painlevé III difference equations
}

\author{
Jilong Zhang ${ }^{1 *}$ and Hongxun $\mathrm{Yi}^{2}$
}

\author{
"Correspondence: \\ jilongzhang2007@gmail.com \\ ${ }^{1}$ LMIB and School of Mathematics \& \\ Systems Science, Beihang \\ University, Beijing, 100191, P.R. China \\ Full list of author information is \\ available at the end of the article
}

\begin{abstract}
In this paper, we investigate the properties of meromorphic solutions of Painlevé III difference equations. In particular, we study the existence of Borel exceptional value, the exponent of convergence of zeros, poles and fixed points of a transcendental meromorphic solution.
\end{abstract}

MSC: 30D35; 39A 10

Keywords: meromorphic solution; difference; finite order

\section{Introduction}

We assume that the reader is familiar with the standard notations and results of Nevanlinna value distribution theory (see, e.g., [1-3]). Let $w$ be a meromorphic function in the complex plane. $\rho(w), \lambda(w)$ and $\lambda(1 / w)$ denote the order, the exponents of convergence of zeros and poles of $w$, respectively. The exponent of convergence of fixed points of $w$ is defined by

$$
\tau(w)=\limsup \frac{\log N\left(r, \frac{1}{w-z}\right)}{\log r} .
$$

Furthermore, we denote by $S(r, w)$ any quantity satisfying $S(r, w)=o(T(r, w))$ for all $r$ outside of a set with finite logarithmic measure and by

$$
\mathcal{S}(w)=\{\alpha \text { meromorphic: } T(r, \alpha)=S(r, w)\}
$$

the field of small functions with respect to $w$. A meromorphic solution $w$ of a difference equation is called admissible if all coefficients of the equation are in $\mathcal{S}(w)$. For example, if a difference equation has only rational coefficients, then all non-rational meromorphic solutions are admissible; if an admissible solution is rational, then all the coefficients must be constants.

Recently, with the development of Nevanlinna value distribution theory on difference expressions [4-6], Halburd and Korhonen [7] considered the difference equation

$$
\bar{w}+\underline{w}=R(z, w)
$$

where $R$ is rational in $w$ and meromorphic in $z$ with slow growth coefficients, and the $z$-dependence is supposed by writing $\bar{w} \equiv w(z+1)$ and $\underline{w} \equiv w(z-1)$. They proved that 
if (1.1) has an admissible meromorphic solution of finite order, then either $w$ satisfies a difference Riccati equation or equation (1.1) can be transformed to eight simple difference equations. These simple difference equations include Painlevé I, II difference equations and linear difference equations.

In 2010, Chen and Shon started the topic of researching the properties of finite-order meromorphic solutions of difference Painlevé I and II equations. In fact, they studied the equations

$$
\begin{aligned}
& \bar{w}+\underline{w}=\frac{\left(a_{1} z+a_{2}\right) w+a_{3}}{1-w^{2}}, \\
& \bar{w}+\underline{w}=\frac{a_{4} z+a_{5}}{w}+a_{6},
\end{aligned}
$$

where $a_{j}(1 \leq j \leq 6)$ are constants with $a_{1} a_{3} a_{4} \neq 0$.

Theorem A ([8]) If $w$ is a transcendental finite-order meromorphic solution of (1.2) or (1.3), then

(i) $w$ has at most one non-zero finite Borel exceptional value;

(ii) $\lambda(1 / w)=\lambda(w)=\tau(w)=\rho(w)$.

Furthermore, assume that a rational function $w=\frac{P(z)}{Q(z)}$ is a solution of $(1.2)$, where $P(z)$ and $Q(z)$ are relatively prime polynomials with degrees $p$ and $q$ respectively, then $q=p+1$, while (1.3) has no rational solution.

As for the family including Painlevé III difference equations, we recall the following theorem.

Theorem B ([9]) Assume that the equation

$$
\bar{w} \underline{w}=R(z, w)
$$

has an admissible meromorphic solution $w$ of hyper-order less than one, where $R(z, w)$ is rational and irreducible in $w$ and meromorphic in $z$, then either $w$ satisfies the difference Riccati equation

$$
\bar{w}=\frac{\alpha w+\beta}{w+\gamma},
$$

where $\alpha, \beta, \gamma \in \mathcal{S}(w)$ are algebroid functions, or equation (1.4) can be transformed to one of the following equations:

$$
\begin{aligned}
& \bar{w} \underline{w}=\frac{\eta w^{2}-\lambda w+\mu}{(w-1)(w-v)}, \\
& \bar{w} \underline{w}=\frac{\eta w^{2}-\lambda w}{(w-1)}, \\
& \bar{w} \underline{w}=\frac{\eta(w-\lambda)}{(w-1)}, \\
& \bar{w} \underline{w}=h w^{m} .
\end{aligned}
$$


In (1.5a), the coefficients satisfy $\kappa^{2} \bar{\mu} \underline{\mu}=\mu^{2}, \bar{\lambda} \mu=\kappa \underline{\lambda} \bar{\mu}, \kappa \overline{\bar{\lambda}} \underline{\lambda}=\underline{\kappa} \lambda \bar{\lambda}$, and one of the following:
(1) $\eta \equiv$
$\bar{v} \underline{v}=1$,
$\kappa=v$;
(2) $\bar{\eta}=\eta=v, \quad \kappa \equiv 1$.

In (1.5b), $\eta \bar{\eta}=1$ and $\overline{\bar{\lambda}} \underline{\lambda}=\lambda \bar{\lambda}$. In (1.5c), the coefficients satisfy one of the following:

(1) $\eta \equiv 1$ and either $\bar{\lambda}=\bar{\lambda} \underline{\lambda}$ or $\bar{\lambda}^{[3]} \underline{\lambda}_{[3]}=\overline{\bar{\lambda}} \underline{\underline{\lambda}}$;

(2) $\bar{\lambda} \underline{\lambda}=\overline{\bar{\lambda}} \underline{\underline{\lambda}}, \bar{\eta} \bar{\lambda}=\overline{\bar{\lambda}} \underline{\eta}, \eta \underline{\eta}=\overline{\bar{\eta}} \underline{\eta}[$;

(3) $\overline{\bar{\eta}} \underline{\eta}=\eta \underline{\eta}, \lambda=\underline{\eta}$;

(4) $\overline{\bar{\lambda}}^{\overline{\overline{[3}}]} \underline{\lambda}_{[3]}=\overline{\bar{\lambda}} \underline{\underline{\lambda}} \lambda, \eta \lambda=\overline{\bar{\eta}} \underline{\underline{\eta}}$.

In $(1.5 \mathrm{~d}), h \in \mathcal{S}(w)$ and $m \in \overline{\bar{Z}},|m| \leq 2$.

The first author and Yang [10] studied the difference Painlevé III equations (1.5b)-(1.5d) with the constant coefficients. In particular, they improved (i) of Theorem A: $w$ does not have Borel exceptional value, and got the following two results.

Theorem C Let $w=\frac{P(z)}{Q(z)}$ be given as in Theorem A. If $w(z)$ is a solution of

$$
\bar{w} \underline{w}(w-1)=\eta(w-\lambda),
$$

where $\eta(\neq 0), \lambda$ are constants, then $p=q$ and $a^{2}(a-1)=\eta(a-\lambda)$, where $a=w(\infty)$.

Theorem D Let $w=\frac{P(z)}{Q(z)}$ be given as in Theorem A. If $w(z)$ is a solution of

$$
\bar{w} \underline{w}(w-1)=w^{2}-\lambda w,
$$

where $\lambda(\neq 1)$ is a constant, then $p=q$ and $a^{2}-2 a+\lambda=0$, where $a=w(\infty)$.

It is natural to ask: What are the properties of a transcendental meromorphic solution $w$ of equations (1.6) and (1.7)? Does $w$ have Borel exceptional value? We will give the answers in Section 3. The remaining equation (1.5a) will be discussed in Section 4.

\section{Some lemmas}

Halburd and Korhonen [5] and Chiang and Feng [4] investigated the value distribution theory of difference expressions. A key result, which is a difference analogue of the logarithmic derivative lemma, reads as follows.

Lemma 2.1 Let $f$ be a meromorphic function offinite order, and let c be a non-zero complex constant. Then

$$
m\left(r, \frac{f(z+c)}{f(z)}\right)+m\left(r, \frac{f(z)}{f(z+c)}\right)=S(r, f) .
$$

With the help of Lemma 2.1, the difference analogues of the Clunie and Mohon'ko lemmas are obtained.

Lemma 2.2 ([6]) Let $f$ be a transcendental meromorphic solution of finite order $\rho$ of a difference equation of the form

$$
U(z, f) P(z, f)=Q(z, f)
$$


where $U(z, f), P(z, f)$ and $Q(z, f)$ are difference polynomials such that the total degree $\operatorname{deg}_{f} U(z, f)=n$ in $f(z)$ and its shifts, and $\operatorname{deg}_{f} Q(z, f) \leq n$. If $U(z, f)$ contains just one term of maximal total degree in $f(z)$ and its shifts, then, for each $\varepsilon>0$,

$$
m(r, P(z, f))=O\left(r^{\rho-1+\varepsilon}\right)+S(r, f),
$$

possibly outside of an exceptional set of finite logarithmic measure.

Lemma 2.3 ([5, 6]) Let $w$ be a transcendental meromorphic solution of finite order of the difference equation

$$
P(z, w)=0,
$$

where $P(z, w)$ is a difference polynomial in $w(z)$. If $P(z, a) \not \equiv 0$ for a meromorphic function $a \in \mathcal{S}(w)$, then

$$
m\left(r, \frac{1}{w-a}\right)=S(r, w)
$$

We conclude this section by the following lemma.

Lemma 2.4 (See, e.g., [3, pp.79-80]) Let $f_{j}(j=1, \ldots, n)(n \geq 2)$ be meromorphic functions, $g_{j}(j=1, \ldots, n)$ be entire functions. If

(i) $\sum_{j=1}^{n} f_{j}(z) e^{g_{j}(z)} \equiv 0$;

(ii) $g_{h}(z)-g_{k}(z)$ is not a constant for $1 \leq h<k \leq n$;

(iii) $T\left(r, f_{j}\right)=S\left(r, e^{g_{h}(z)-g_{k}(z)}\right)$ for $1 \leq j \leq n$ and $1 \leq h<k \leq n$, then $f_{j}(z) \equiv 0(j=1, \ldots, n)$.

\section{Equations (1.6) and (1.7)}

Theorem 3.1 If $w$ is a transcendental finite-order meromorphic solution of (1.6), then

(i) $\lambda(w)=\tau(w)=\rho(w)$;

(ii) If $\lambda=0$, then $w$ has at most one non-zero Borel exceptional value for $\rho(w)>0$.

Proof Denote $\phi(z)=w(z)-z$. So, $\phi(z)$ is a transcendental meromorphic function and $T(r, \phi)=T(r, w)+S(r, w)$. Substituting $w(z)=\phi(z)+z$ in (1.6), we obtain

$$
(\bar{\phi}+z+1)(\phi+z-1)(\phi+z-1)=\eta(\phi+z-\lambda) .
$$

Let

$$
P(z, \phi)=(\bar{\phi}+z+1)(\underline{\phi}+z-1)(\phi+z-1)-\eta(\phi+z-\lambda) .
$$

We get $P(z, 0)=(z-1)^{2}(z+1)-\eta(z-\lambda) \not \equiv 0$, Lemma 2.3 gives

$$
m\left(r, \frac{1}{w-z}\right)=m(r, 1 / \phi)=S(r, \phi)
$$

which means $N\left(r, \frac{1}{w-z}\right)=T(r, w)+S(r, w)$, and thus $\tau(w)=\rho(w)$. 
If $\lambda \neq 0$, Lemma 2.3 tells us $m(r, 1 / w)=S(r, w)$. If $\lambda=0$, we rewrite (1.6) as

$$
\frac{\bar{w}}{w} \frac{\underline{w}}{w} w-\frac{\bar{w}}{w} \frac{\underline{w}}{w}=\eta \frac{1}{w}
$$

Noting that $m(r, w)=S(r, w)$, by applying Lemma 2.2 to (1.6), we deduce from Lemma 2.1 and the above equation that

$$
m(r, 1 / w)=S(r, w)
$$

then $\lambda(w)=\rho(w)$ holds.

Next, we prove the conclusion (ii). Some ideas here come from [8]. Assume to the contrary that $w$ has two non-zero Borel exceptional values $a$ and $b(\neq a)$. Set

$$
f(z)=\frac{w(z)-a}{w(z)-b} .
$$

Then $\rho(f)=\rho(w), \lambda(f)=\lambda(w-a)<\rho(f)$ and $\lambda(1 / f)=\lambda(w-b)<\rho(f)$. Since $f$ is of finite order, we suppose that

$$
f(z)=g(z) e^{d z^{n}}
$$

where $d(\neq 0)$ is a constant, $n(\geq 1)$ is an integer, $g(z)$ is meromorphic and satisfies

$$
\rho(g)<\rho(f)=n
$$

Then

$$
f(z+1)=g(z+1) g_{1}(z) e^{d z^{n}}, \quad f(z-1)=g(z-1) g_{2}(z) e^{d z^{n}}
$$

where $g_{1}(z)=e^{n d z^{n-1}+\cdots+d}$ and $g_{2}(z)=e^{-n d z^{n-1}+\cdots+(-1)^{n} d}$.

We get from (3.1) and (3.2) that $w=\frac{b f-a}{f-1}$. Noting that $\lambda=0$, by (1.6) and (3.4), we have

$$
A(z) e^{3 d z^{n}}+B(z) e^{2 d z^{n}}+C(z) e^{d z^{n}}+D=0
$$

where

$$
\begin{aligned}
& A(z)=\left[b^{2}(b-1)-\eta b\right] g \bar{g} g_{1} g g_{2}, \\
& B(z)=\left[b^{2}(1-a)+\eta a\right] \bar{g} g_{1} \underline{g} g_{2}+[b \eta+a b(1-b)] g\left(\bar{g} g_{1}+\underline{g} g_{2}\right), \\
& C(z)=\left[a^{2}(b-1)-\eta b\right] g+[a b(a-1)-\eta a]\left(\bar{g} g_{1}+g g_{2}\right), \\
& D=a^{2}(1-a)+\eta a .
\end{aligned}
$$

From (3.3), we apply Lemma 2.4 on (3.5), resulting in vanishing of all the coefficients. Since $a$ and $b$ are non-zero constants, we deduce from $A(z)=0$ and $D=0$ that

$$
a(a-1)=\eta, \quad b(b-1)=\eta,
$$


which means that $a$ and $b$ are distinct zeros of the equation

$$
z^{2}-z-\eta=0
$$

Thus

$$
a+b=1, \quad a b=-\eta .
$$

Denote $G=g, G_{1}=\bar{g} g_{1}$ and $G_{2}=g g_{2}$. From $B(z)=0, C(z)=0$ and (3.6), we have

$$
\begin{aligned}
& (a-1)(a+b) G_{1} G_{2}=b(b-1) G\left(G_{1}+G_{2}\right), \\
& (b-1)(a+b) G=a(a-1)\left(G_{1}+G_{2}\right) .
\end{aligned}
$$

It follows from (3.7) that

$$
\begin{aligned}
& G_{1} G_{2}=a G\left(G_{1}+G_{2}\right), \\
& G=b\left(G_{1}+G_{2}\right) .
\end{aligned}
$$

Since the last two equations are both homogeneous, there exist two non-zero constants $\alpha$ and $\beta$ such that $G_{1}=\alpha G$ and $G_{2}=\beta G$. Then

$$
\alpha \beta=\frac{a}{b}
$$

On the other hand, combining (3.2) with (3.4) yields

$$
\bar{f}=\alpha f, \quad \underline{f}=\beta f,
$$

which yield $\alpha \beta=1$. Thus $a=b$ by (3.8), a contradiction.

Theorem 3.2 If $w$ is a transcendental meromorphic solution of (1.7) with finite order $\rho(w)>0$, then

(i) $\tau(w)=\rho(w)$

(ii) If $\lambda \neq 0$, then $\lambda(w)=\rho(w)$;

(iii) $w$ has at most one non-zero Borel exceptional value.

Proof The conclusions (i) and (ii) can be discussed by the same reasoning as in the proof of Theorem 3.1, we only prove the conclusion (iii) here. Assume to the contrary that $w$ has two non-zero Borel exceptional values $a$ and $b(\neq a)$. Let $f$ be given by (3.1). Then we still have (3.2)-(3.4). Substituting $w=\frac{b f-a}{f-1}$ in (1.7), we obtain

$$
A_{1}(z) e^{4 d z^{n}}+B_{1}(z) e^{3 d z^{n}}+C_{1}(z) e^{2 d z^{n}}+D_{1}(z) e^{d z^{n}}+E_{1}=0,
$$

where

$$
\begin{aligned}
& A_{1}(z)=\left[b^{2}(b-1)-b(b-\lambda)\right] g^{2} \bar{g} g_{1} g g_{2}, \\
& B_{1}(z)=\left[b^{2}(2-a-b)+2 a b-(a+b) \lambda\right] g \bar{g} g_{1} g g_{2}+[b(b-\lambda)-a b(b-1)] g^{2}\left(\bar{g} g_{1}+g g_{2}\right),
\end{aligned}
$$




$$
\begin{aligned}
& C_{1}(z)= {\left[b^{2}(a-1)-a(a-\lambda)\right] \bar{g} g_{1} \underline{g} g_{2}+\left[a^{2}(b-1)-b(b-\lambda)\right] g^{2} } \\
&+[a b(a+b-2)+(a+b) \lambda-2 a b] g\left(\bar{g} g_{1}+\underline{g} g_{2}\right), \\
& D_{1}(z)= {[a b(1-a)+a(a-\lambda)]\left(\bar{g} g_{1}+g g_{2}\right) } \\
&+ {\left[a^{2}(2-a-b)+2 a b-(a+b) \lambda\right] g, } \\
& E_{1}=a^{2}(a-1)-a(a-\lambda) .
\end{aligned}
$$

From (3.3), we apply Lemma 2.4 to (3.9), which results in vanishing of all the coefficients. By a similar way to that above, we deduce from $A_{1}(z)=0$ and $E_{1}=0$ that $a$ and $b$ are distinct zeros of the equation

$$
z^{2}-2 z+\lambda=0
$$

Thus

$$
a+b=2, \quad a b=\lambda .
$$

Noting this, from $B_{1}(z)=0$, we have $b(b-\lambda)-a b(b-1)=0$ or $\bar{g} g_{1}+g g_{2}=0$. If $b(b-\lambda)-$ $a b(b-1)=0$, then (3.10) gives $\lambda=1$, a contradiction. Therefore, $\bar{g} g_{1}+\underline{g} g_{2}=0$, i.e.,

$$
\bar{f}=-f .
$$

We get from $\bar{g} g_{1}+g g_{2}=0$ and $C_{1}(z)=0$ that

$$
\left(a b^{2}-a^{2}-b^{2}+a \lambda\right)\left(\bar{f} f+f^{2}\right)=0 .
$$

If $a b^{2}-a^{2}-b^{2}+a \lambda=0$, we have from (3.10) that $a=b$, which is a contradiction. Then $\bar{f} f+f^{2}=0,(3.11)$ yields

$$
\bar{f}= \pm f
$$

Combining (3.11) and the last equation gives a contradiction, and (iii) follows.

\section{Equation (1.5a)}

Assume that the coefficients are constants in (1.5a). In this section, we consider the equation

$$
\bar{w} \underline{w}(w-1)^{2}=w^{2}-\lambda w+\mu,
$$

where $\lambda$ and $\mu$ are constants.

Theorem 4.1 Let $w=\frac{P(z)}{Q(z)}$ be given as in Theorem A. If $w(z)$ is a solution of (4.1), then one of the following holds:

(i) $p=q, a^{2}(a-1)^{2}=a^{2}-\lambda a+\mu$, where $a=w(\infty)$;

(ii) $p<q, \lambda=\mu=0$ and $P(z)$ is a constant. 
Proof Substituting $w$ by $\frac{P(z)}{Q(z)}$ in (4.1), we get

$$
\frac{P(z+1)}{Q(z+1)} \frac{P(z-1)}{Q(z-1)}\left(\frac{P(z)}{Q(z)}-1\right)^{2}=\left(\frac{P(z)}{Q(z)}\right)^{2}-\lambda \frac{P(z)}{Q(z)}+\mu .
$$

Let $s=p-q$. We discuss the following three cases.

Case 1. $s>0$. Then $\frac{P(z)}{Q(z)}=a z^{s}(1+o(1))$ as $r$ tends to infinite, and (4.2) gives

$$
a^{2}(z+1)^{s}(z-1)^{s}(1+o(1))\left(a z^{s}(1+o(1))-1\right)^{2}=a^{2} z^{2 s}(1+o(1))
$$

which is a contradiction as $r$ tends to infinite.

Case 2. $s<0$. Now, $\frac{P(z)}{Q(z)}=o(1)$ and $\frac{P(z+1)}{Q(z+1)}=o(1)$. By (4.2), we obtain $\mu=0$. If $\lambda \neq 0$, equation (4.2) turns into

$$
\left(\frac{Q(z)}{P(z)}-1\right)^{2}=\left(1-\lambda \frac{Q(z)}{P(z)}\right) \frac{Q(z+1)}{P(z+1)} \frac{Q(z-1)}{P(z-1)} .
$$

Noting that $\frac{Q(z)}{P(z)}=b z^{-S}(1+o(1))$ as $r$ tends to infinite, the above equation also leads to a contradiction by the same reasoning as in Case 1.

Then $\lambda=0$. We have

$$
\frac{Q(z+1)}{P(z+1)} \frac{Q(z-1)}{P(z-1)}=\frac{(Q(z)-P(z))^{2}}{P^{2}(z)} .
$$

It is easy to find that $P^{2}(z)$ and $P(z+1) P(z-1)$ have the same zeros, which means that $P(z)$ must be a constant. We get (ii).

Case 3. $s=0$. We suppose that $\frac{P(z)}{Q(z)}=a+o(1)$ as $r$ tends to infinite. The conclusion (i) follows from (4.2).

Example 4.2 The rational function $w(z)=\frac{1}{(z+1)^{2}}$ is a solution of the difference equation $\bar{w} \underline{w}(w-1)^{2}=w^{2}$. This shows that the conclusion (ii) of Theorem 4.1 may occur.

By the same reasoning as in Section 3, we obtain the following result.

Theorem 4.3 If $w$ is a transcendental meromorphic solution of (4.1) with finite order $\rho(w)$, then

(i) $\tau(w)=\rho(w)$;

(ii) If $\lambda \mu \neq 0$, then $\lambda(w)=\rho(w)$.

Example 4.4 The function $w(z)=\sec ^{2} \frac{\pi z}{2}$ is a solution of the difference equation $\bar{w} \underline{w}(w-$ $1)^{2}=w^{2} .0$ is a Picard exceptional value of $w$, which shows that $\lambda \mu \neq 0$ is necessary in (ii) of Theorem 4.3. 


\section{Author details}

${ }^{1}$ LMIB and School of Mathematics \& Systems Science, Beihang University, Beijing, 100191, P.R. China. ${ }^{2}$ School of Mathematics, Shandong University, Jinan, Shandong 250100, P.R. China.

\section{Acknowledgements}

The authors would like to thank the referee for valuable suggestions to the present paper. This research was supported by the NNSF of China no. 11201014, 11171013, 11126036 and the YWF-ZY-302854 of Beihang University. This research was also supported by the youth talent program of Beijing.

Received: 17 June 2013 Accepted: 6 August 2013 Published: 21 August 2013

\section{References}

1. Hayman, WK: Meromorphic Functions. Clarendon, Oxford (1964)

2. Laine, I: Nevanlinna Theory and Complex Differential Equations. de Gruyter, Berlin (1993)

3. Yang, CC, Yi, HX: Uniqueness Theory of Meromorphic Functions. Kluwer Academic, Dordrecht (2003)

4. Chiang, YM, Feng, SJ: On the Nevanlinna characteristic of $f(z+\eta)$ and difference equations in the complex plane. Ramanujan J. 16, 105-129(2008)

5. Halburd, RG, Korhonen, RJ: Difference analogue of the lemma on the logarithmic derivative with applications to difference equations. J. Math. Anal. Appl. 314, 477-487 (2006)

6. Laine, I, Yang, CC: Clunie theorems for difference and q-difference polynomials. J. Lond. Math. Soc. 76, 556-566 (2007)

7. Halburd, RG, Korhonen, RJ: Finite order solutions and the discrete Painlevé equations. Proc. Lond. Math. Soc. 94, 443-474 (2007)

8. Chen, ZX, Shon, $\mathrm{KH}$ : Value distribution of meromorphic solutions of certain difference Painlevé equations. J. Math Anal. Appl. 364, 556-566 (2010)

9. Ronkainen, O: Meromorphic solutions of difference Painlevé equations. Ann. Acad. Sci. Fenn. Diss. 155 (2010), $59 \mathrm{pp}$

10. Zhang, JL, Yang, LZ: Meromorphic solutions of Painlevé III difference equations. Acta Math. Sin. (to appear)

doi:10.1186/1687-1847-2013-256

Cite this article as: Zhang and Yi: Properties of meromorphic solutions of Painlevé III difference equations. Advances in Difference Equations 2013 2013:256.

\section{Submit your manuscript to a SpringerOpen ${ }^{\circ}$ journal and benefit from:}

- Convenient online submission

Rigorous peer review

- Immediate publication on acceptance

Open access: articles freely available online

- High visibility within the field

- Retaining the copyright to your article 